\title{
Attenuation Correction Maps for SPECT Myocardial Perfusion Imaging from Contrast-Enhanced Coronary CT Angiography: Gemstone Spectral Imaging with Single-Source Dual Energy and Material Decomposition
}

\author{
Tobias A. Fuchs*1, Bert-Ram Sah*1, Julia Stehli ${ }^{1}$, Sacha Bull ${ }^{1}$, Svetlana Dougoud ${ }^{1}$, Martin W. Huellner ${ }^{1}$, \\ Oliver Gaemperli ${ }^{1}$, and Philipp A. Kaufmann ${ }^{1,2}$ \\ ${ }^{\text {I}}$ Cardiac Imaging, Department of Radiology, University Hospital Zurich, Zurich, Switzerland; and ${ }^{2}$ Zurich Center for Integrative \\ Human Physiology (ZIHP), University of Zurich, Zurich, Switzerland
}

\begin{abstract}
The aim of this study was to explore the feasibility of attenuation correction (AC) of myocardial perfusion imaging (MPI) with a virtual unenhanced cardiac CT scan synthesized from contrast-enhanced single-source dual-energy coronary CT angiography. Methods: Segmental myocardial percentage uptake values obtained with $\mathrm{AC}$ were analyzed by use of correlation analysis and Bland-Altman limits of agreement (20-segment model), and clinical agreement was evaluated in 30 patients. Results: The 2 methods showed an excellent correlation for segmental myocardial percentage uptake at stress $(r=0.93 ; P<0.001$; low dose) and at rest $(r=0.90 ; P<$ 0.001 ; high dose) with narrow Bland-Altman limits of agreement ( $-6.8 \%$ to $7.8 \%$ and $-7.8 \%$ to $7.4 \%$, respectively). The levels of clinical agreement of SPECT MPI corrected with standard versus virtual unenhanced CT AC were $99 \%$ per coronary territory and 97\% per patient. Conclusion: Our results suggest that $A C$ of SPECT MPI with a virtual unenhanced CT scan synthesized from contrast-enhanced coronary CT angiography is feasible and reliable.
\end{abstract}

Key Words: virtual unenhanced CT; attenuation correction; SPECT; gemstone spectral imaging

J Nucl Med 2013; 54:2077-2080

DOI: 10.2967/jnumed.113.123570

$\mathbf{U}$ nenhanced cardiac CT for attenuation correction (AC) has been shown to improve the image quality as well as the diagnostic and prognostic accuracy ( 1 ) of SPECT myocardial perfusion imaging (MPI) for the evaluation of coronary artery disease (CAD).

Because the integration of coronary CT angiography (CCTA) and SPECT MPI into a single fused hybrid image has been shown to further improve diagnostic (2) and prognostic (3) assessments of CAD, cardiac hybrid imaging has been suggested as a gatekeeper for invasive coronary angiography $(4,5)$.

\footnotetext{
Received Mar. 22, 2013; revision accepted Sep. 4, 2013.

For correspondence or reprints contact: Philipp A. Kaufmann, Cardiac Imaging, Department of Radiology, University Hospital Zurich, Ramistrasse 100, NUK C 42, CH-8091 Zurich, Switzerland.

E-mail: pak@usz.ch

${ }^{*}$ Contributed equally to this work.

Published online Oct. 21, 2013.

COPYRIGHT (C) 2013 by the Society of Nuclear Medicine and Molecular Imaging, Inc.
}

Although the introduction of prospective electrocardiogramtriggered low-dose CCTA and improvements in SPECT MPI have reduced radiation dose exposure from initially about $40 \mathrm{mSv}$ to 3-5 $\mathrm{mSv}(6)$, any further dose reduction in hybrid cardiac imaging is welcome. So far, an additional unenhanced CT scan is required for SPECT AC in cardiac hybrid SPECT/CT imaging because CCTA cannot be used for AC. With the introduction of dual-energy scanning with dual-source (7) or single-source (8) CT scanners, material decomposition, particularly iodine subtraction, has become available. This methodology allows virtual unenhanced CT to be obtained from contrast-enhanced CCTA.

The aim of the present pilot study was to evaluate whether virtual unenhanced images generated from contrast-enhanced singlesource dual-energy CCTA can be used for AC of myocardial SPECT.

\section{MATERIALS AND METHODS}

\section{Study Population}

Thirty consecutive patients referred for the evaluation of known or suspected CAD underwent SPECT MPI with unenhanced CT for AC and contrast-enhanced CCTA $(1,3,6)$. The need for written informed consent was waived by the institutional review board (local ethics committee) because of the retrospective nature of this study with solely clinical data collection.

\section{Image Acquisition and Analysis}

The 1-d ${ }^{99 \mathrm{~m}} \mathrm{Tc}$-tetrofosmin adenosine stress $(0.14 \mathrm{mg} / \mathrm{kg} / \mathrm{min}$ over $6 \mathrm{~min}) /$ rest electrocardiogram-gated imaging protocol was previously described in detail (9-11). In brief, $60 \mathrm{~min}$ after the injection of 300$350 \mathrm{MBq}$ of ${ }^{99 \mathrm{~m}} \mathrm{Tc}$-tetrofosmin, images were acquired (over $5 \mathrm{~min}$ ) with a cadmium-zinc-telluride camera, a tracer dose 3 times higher than the stress dose was injected, and image acquisition was initiated within a few minutes.

SPECT MPI scans were reconstructed in standard axes (short axis, vertical long axis, and horizontal long axis), and polar maps of perfusion were generated with a 20 -segment left ventricular model by use of a commercially available software package (Cedars QGS/QPS; Cedars-Sinai Medical Center). Segmental uptake as a percentage of maximum uptake and visual (3) and quantitative summed rest scores (SRS) and summed stress scores (SSS) (12) obtained with standard unenhanced CT AC (13) were compared with the values obtained with virtual unenhanced CT AC (Fig. 1).

In addition, the presence or absence of perfusion defects in the 3 main coronary territories-left anterior descending artery, circumflex 

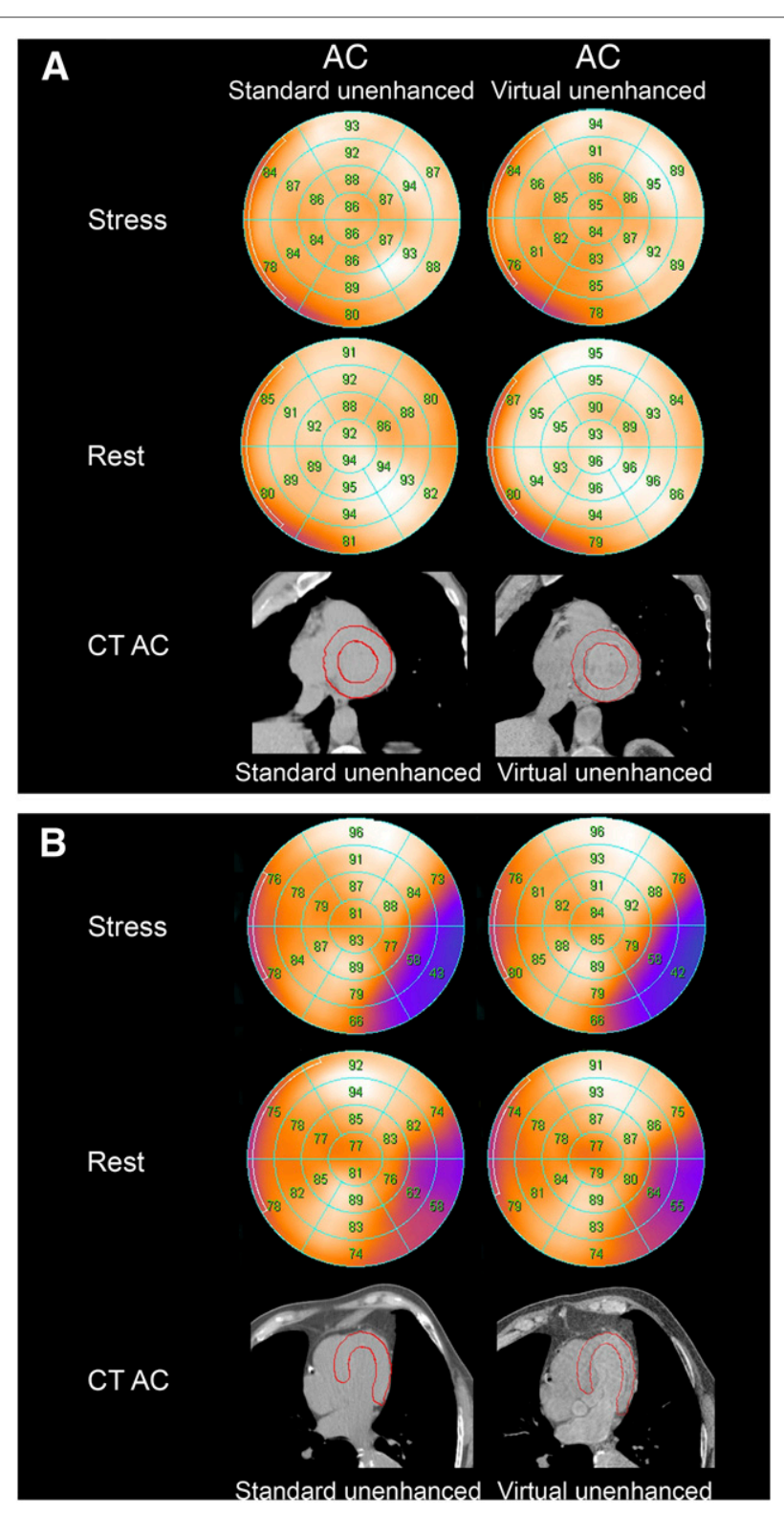

FIGURE 1. MPI polar plots with normal perfusion (A) and with partially reversible inferolateral perfusion defect $(B)$. Findings with standard unenhanced CT AC (left) and virtual unenhanced CT AC (right) were identical.

artery, and right coronary artery—was evaluated without knowledge of the AC method.

Metoprolol (up to $25 \mathrm{mg}$; Beloc; AstraZeneca) was administered intravenously before CT scanning if the heart rate was higher than 65 beats per minute (6), and all patients received $2.5 \mathrm{mg}$ of sublingual isosorbide dinitrate (Isoket; Schwarz Pharma).

Unenhanced whole-heart CT scans were acquired during inspiration breath hold with the following parameters: prospective electrocardiogram-triggered detector coverage, $40 \mathrm{~mm}$; slice thickness, 2.5 $\mathrm{mm}$; gantry rotation time, $0.35 \mathrm{~s}$; temporal resolution, $0.175 \mathrm{~s}$; tube voltage, $120 \mathrm{kVp}$; and tube current, $200 \mathrm{~mA}$. CT images were reconstructed with a section thickness of $5.0 \mathrm{~mm}$ and a field of view of $50 \mathrm{~cm}$ and were transferred to a Xeleris workstation (GE Healthcare) for the generation of AC maps (14).

CCTA parameters were as follows: body surface-adapted iodixanol (320 mg/mL; Visipaque 320; GE Healthcare) administration (15);
$64 \times 0.625 \mathrm{~mm}$; gantry rotation time, $0.35 \mathrm{~s}$; temporal resolution, $0.175 \mathrm{~s}$; and body mass index-adapted tube current and mean voltage (by switching within $0.5 \mathrm{~ms}$ between 80 and $140 \mathrm{kVp}$ as previously reported (8)). The beam was kept at $140 \mathrm{kVp}$ only for a fraction (about one-third) of the radiation time to keep the radiation dose as low as that reported in standard low-dose CCTA (8). Decomposition of preselected materials can be achieved by selecting adequate triplets from the material library. With this technique, a contrast agent can be removed from a dual-energy examination, producing a virtual unenhanced image (16) within seconds. Monochromatic CT images (at $70 \mathrm{keV}$ ) were reconstructed with a slice thickness of $0.625 \mathrm{~mm}$ for iodine subtraction on a dedicated workstation and transferred to a Xeleris workstation for the generation of a virtual unenhanced AC map. Hounsfield units (HU) were measured in the left ventricle, the lungs, and the vertebrae with both CT AC methods (Fig. 2).

The effective dose of cardiac CT was calculated as the dose-length product times a conversion coefficient for the chest: $\mathrm{k}=0.014 \mathrm{mSv} /$ $(\mathrm{mGy} \times \mathrm{cm})(17)$. For SPECT, the effective radiation dose was estimated as previously suggested $(6.7 \mathrm{mSv} / \mathrm{GBq})(6)$.

\section{Statistical Analysis}

Quantitative variables were expressed as mean $\pm \mathrm{SD}$, and categorical variables were expressed as frequencies, means, or percentages. The data were tested with SPSS software (version 20.0; SPSS Inc.) by use of the Shapiro-Wilk test for a normal distribution and the Wilcoxon signed rank test for a nonnormal distribution. Results from standard unenhanced $\mathrm{AC}$ were compared with results from virtual unenhanced AC by Pearson correlation, and Bland-Altman (BA) limits of agreement were calculated. $P$ values of less than 0.05 were considered statistically significant.

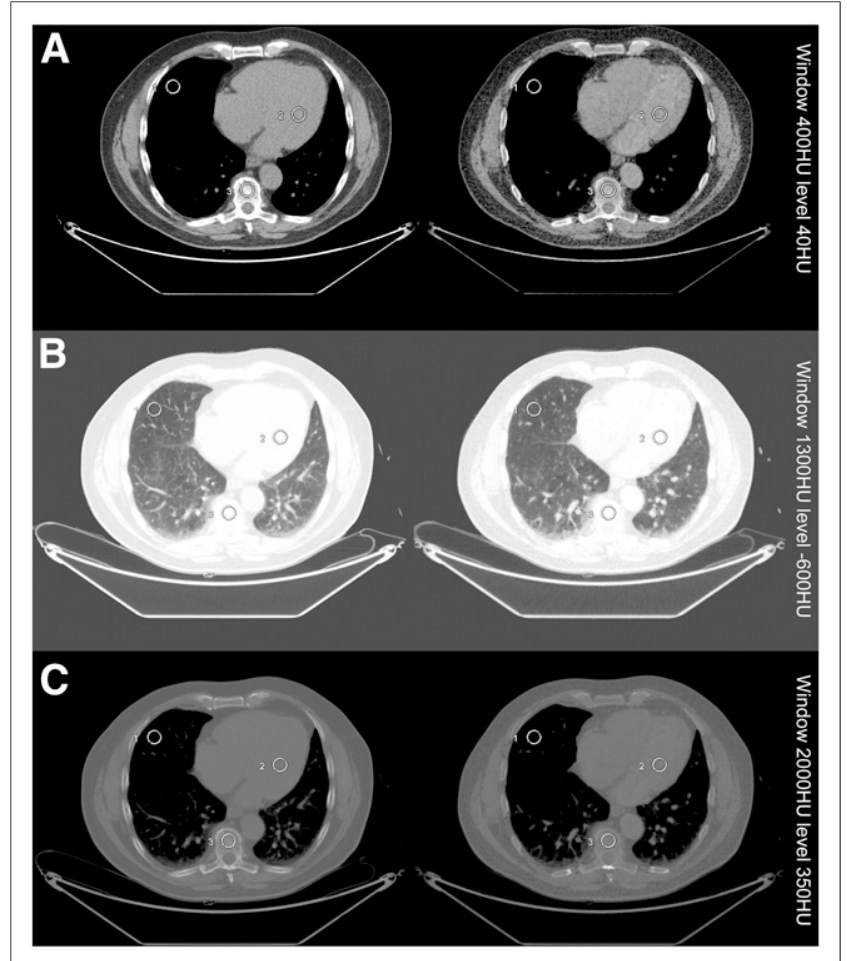

FIGURE 2. Analysis of standard unenhanced CT (left) and virtual unenhanced $C T$ (right) with soft-tissue (A), lung $(B)$, and bone (C) windows revealed comparable $\mathrm{HU}$ in lung (ROI 1: -877 and -878 , respectively), left ventricular (ROI 2: 41 and 46, respectively), and bone (ROI 3: 168 and 87 , respectively) tissues. 


\section{RESULTS}

All 30 patients successfully underwent the protocol. Their characteristics are shown in Table 1 . The radiation doses (mean \pm SD) were $2.3 \pm 0.3 \mathrm{mSv}$ for stress MPI, $6.6 \pm 0.8 \mathrm{mSv}$ for rest MPI, $0.8 \pm 0.0 \mathrm{mSv}$ for unenhanced cardiac CT, and $1.6 \pm 0.4 \mathrm{mSv}$ for contrast-enhanced CCTA.

The BA limits of attenuation agreement between the 2 unenhanced CT scans were -28 to $20 \mathrm{HU}$ for the left ventricle (mean, $-4 \mathrm{HU}$ ), -10 to $20 \mathrm{HU}$ for the lungs (mean, $5 \mathrm{HU}$ ), and 38 to 120 $\mathrm{HU}$ for the bones (mean, $79 \mathrm{HU}$ ).

In 600 segments (20 segments in 30 patients), a comparison of tracer uptake in standard unenhanced CT AC and that in virtual unenhanced CT AC revealed an excellent correlation at stress $(r=$ 0.93; $P<0.001$; low dose) and at rest $(r=0.90 ; P<0.001$; high dose) with narrow BA limits of agreement $(-6.8 \%$ to $7.8 \%$ and $-7.8 \%$ to $7.4 \%$, respectively) (Fig. 3).

Of the 90 possible coronary territories ( 3 coronary territories in 30 patients), 10 had a perfusion defect at stress and 7 had a perfusion defect at rest after reconstruction with standard CT AC. Virtual unenhanced CT AC revealed 9 perfusion defects at stress and 8 perfusion defects at rest, for a stress-rest per-territory agreement of $99 \%$ (Fig. 4). Standard AC and virtual AC revealed pathology (scar or ischemia) in 9 and 8 patients, respectively, for a $97 \%$ overall clinical agreement. The BA limits of agreement for SRS and SSS were narrow for visual (SRS, -1.0 to 0.9 ; SSS, -1.1 to 1.3 ) and automated (SRS, -2.8 to 2.6 ; SSS, -2.3 to 2.5 ) evaluations.

\section{DISCUSSION}

The present study demonstrated for, to our knowledge, the first time the feasibility of obtaining accurate AC maps from virtual unenhanced CT generated from contrast-enhanced CCTA by subtracting iodine through material decomposition. This result was achieved with single-source dual-energy CT acquisition at different $\mathrm{x}$-ray energy levels, fast kilovolt peak switching, and the latest gemstone detectors; these techniques allowed the selective suppression of materials, especially those with high atomic

TABLE 1

Characteristics of 30 Patients

\begin{tabular}{|cc|}
\hline \multicolumn{1}{c}{ Characteristic } & Value \\
\hline Age (years, mean $\pm \mathrm{SD})$ & $66.1 \pm 8.3$ \\
\hline Men & $18(60)$ \\
\hline Body mass index $\left(\mathrm{kg} / \mathrm{m}^{2}\right.$, mean $\left.\pm \mathrm{SD}\right)$ & $24.7 \pm 3.6$ \\
\hline Cardiovascular risk factors & $16(53)$ \\
\hline Hypertension & $18(60)$ \\
\hline Dyslipidemia & $14(47)$ \\
\hline Smoking & $12(40)$ \\
\hline Positive family history & $2(7)$ \\
\hline Diabetes & $4(13)$ \\
\hline History of CAD & $7(23)$ \\
\hline Single-vessel CAD & $5(17)$ \\
\hline Multivessel CAD & $8(27)$ \\
\hline Myocardial infarction & $7(23)$ \\
\hline Invasive coronary angiography & $1(3)$ \\
\hline Percutaneous coronary intervention &
\end{tabular}

Values are reported as number of patients followed by percentage in parentheses unless otherwise indicated.

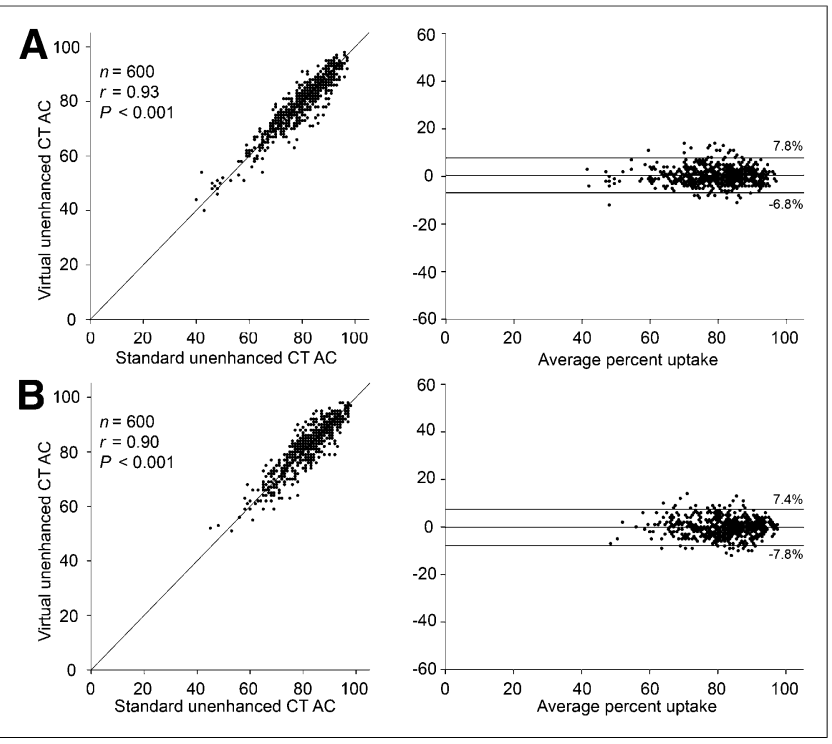

FIGURE 3. Comparison of quantitative percent segmental tracer uptake with standard unenhanced CT AC and virtual unenhanced CT AC revealed excellent correlation coefficients at stress (low dose) $(A)$ and at rest (high dose) $(\mathrm{B})$ with narrow $\mathrm{BA}$ limits of agreement.

numbers, such as iodine, for the generation of virtual unenhanced images.

The SPECT images obtained with virtual unenhanced CT AC showed reliable clinical findings and conclusions when compared with SPECT MPI obtained with standard unenhanced CT AC. Because this method may minimize scan time as well as radiation exposure and improve cost-effectiveness because it renders an unenhanced CT scan unnecessary, it may have the potential to emerge as an alternative to standard unenhanced CT AC in patients undergoing hybrid imaging. This notion is further supported by the excellent correlation of the segmental percentage tracer uptake values obtained from the $2 \mathrm{AC}$ methods with narrow BA limits of agreement, largely corresponding to those obtained from a test-retest validation with the QGS/QPS tool (12) in the present study; these data suggest that a true difference between synthetic CT AC and standard CT AT seems almost negligible.

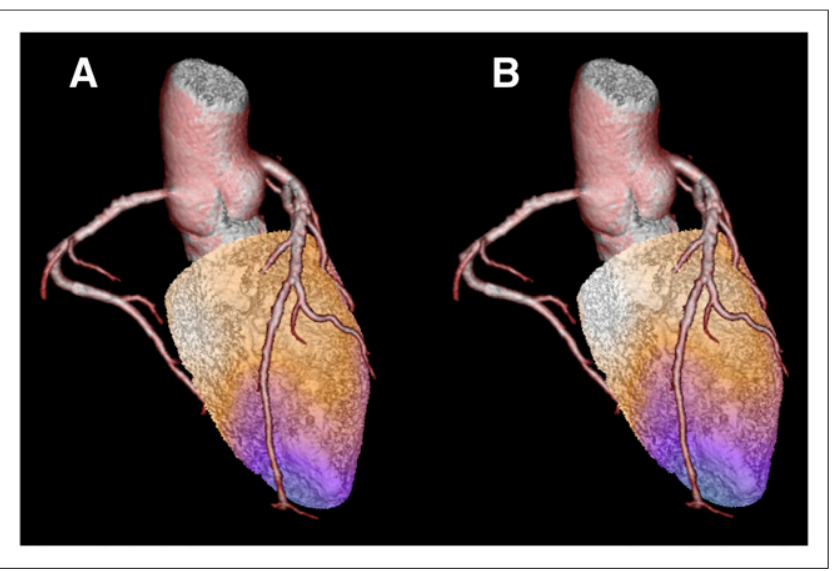

FIGURE 4. Hybrid cardiac SPECT/CCTA with standard unenhanced CT AC (A) and virtual unenhanced CT AC (B) revealed same perfusion defect in left anterior descending artery territory (pink). 
Several methods have been proposed for AC of MPI. Initially, attenuation maps were most commonly based on radionuclide line sources $(2,3)$, and then CT-based AC was introduced. Despite the advent of the latest cardiac $\gamma$-camera generation with semiconductor detectors and new detector array geometry, the problems of nonuniform soft-tissue attenuation have not been solved. The diagnostic accuracy of SPECT MPI is still adversely affected by attenuation artifacts but can be improved through the use of CT-based AC maps (13). In addition, improved prognostic prediction has been reported through the use of attenuation-corrected versus uncorrected SPECT MPI (1), further supporting the important role of appropriate AC.

The implementation of the method described here is particularly pertinent in view of the emerging use of hybrid cardiac imaging, which has been promoted by the integration of SPECT and PET scanners with high-end CT devices into hybrid scanners. This configuration allows the integration of information on anatomy (CCTA) with perfusion images. By revealing both the burden of anatomic $\mathrm{CAD}$ and its pathophysiologic relevance, hybrid imaging can provide unique information that may increase the noninvasive diagnosis, risk assessment, and management of CAD (5).

In its infancy, hybrid cardiac imaging with CCTA and SPECT MPI was associated with exposures of patients to excessively high levels of radiation (up to $40 \mathrm{mSv}$ ). The introduction of prospective triggering (18) and modern SPECT MPI protocols (11) has reduced radiation exposures in patients undergoing hybrid imaging to $3-5 \mathrm{mSv}$. It is in this context that a radiation exposure reduction of $0.8 \mathrm{mSv}$ must be seen as another important step on the journey to minimizing the radiation dose to as low as reasonably achievable (ALARA), according to the ALARA principle for radiation protection. Thus, any method that further reduces radiation exposure is welcome and should be endorsed.

A potential limitation of the present study was that the slice thicknesses of the acquired and reconstructed images differed between the standard and virtual unenhanced scans, as the latter were obtained from CCTA, which has a high resolution. However, because SPECT images have a lower resolution-by about 1 order of magnitude - it appears reasonable to assume that these differences may not have interfered with our results, as the attenuation maps were smoothed to equate their resolution with that of the emission images (19). Furthermore, although our results documented successful AC over a large range of body mass indices $\left(17.9-34.1 \mathrm{~kg} / \mathrm{m}^{2}\right)$, extrapolation to patients with body mass indices beyond this range must be done with caution because it requires confirmation, particularly with higher body mass indices and larger study populations. Finally, we did not use invasive coronary angiography as a standard of reference and therefore cannot entirely exclude overcorrection of true inferior artifacts. However, we believed that it would be more appropriate to use the same technique, namely, MPI with $\mathrm{AC}$, as the standard of reference rather than an anatomic reference, particularly because the accuracy of CT AC for SPECT has been well established (13).

\section{CONCLUSION}

Our results suggest that AC of SPECT MPI with a virtual unenhanced CT scan synthesized from contrast-enhanced CCTA is feasible and reliable.

\section{DISCLOSURE}

The costs of publication of this article were defrayed in part by the payment of page charges. Therefore, and solely to indicate this fact, this article is hereby marked "advertisement" in accordance with 18 USC section 1734. This study was supported in part by grants from the Swiss National Science Foundation. No other potential conflict of interest relevant to this article was reported.

\section{ACKNOWLEDGMENTS}

We thank Ennio Mueller and Gentian Cermjani for their excellent technical support.

\section{REFERENCES}

1. Pazhenkottil AP, Ghadri JR, Nkoulou RN, et al. Improved outcome prediction by SPECT myocardial perfusion imaging after CT attenuation correction. $\mathrm{J} \mathrm{Nucl}$ Med. 2011;52:196-200.

2. Gaemperli O, Schepis T, Valenta I, et al. Cardiac image fusion from stand-alone SPECT and CT: clinical experience. J Nucl Med. 2007;48:696-703.

3. Pazhenkottil AP, Nkoulou RN, Ghadri JR, et al. Prognostic value of cardiac hybrid imaging integrating single-photon emission computed tomography with coronary computed tomography angiography. Eur Heart J. 2011;32:1465-1471.

4. Gaemperli O, Husmann L, Schepis T, et al. Coronary CT angiography and myocardial perfusion imaging to detect flow-limiting stenoses: a potential gatekeeper for coronary revascularization? Eur Heart J. 2009;30:2921-2929.

5. Pazhenkottil AP, Nkoulou RN, Ghadri JR, et al. Impact of cardiac hybrid singlephoton emission computed tomography/computed tomography imaging on choice of treatment strategy in coronary artery disease. Eur Heart J. 2011;32:2824-2829.

6. Husmann L, Herzog BA, Gaemperli O, et al. Diagnostic accuracy of computed tomography coronary angiography and evaluation of stress-only single-photon emission computed tomography/computed tomography hybrid imaging: comparison of prospective electrocardiogram-triggering vs. retrospective gating. Eur Heart J. 2009;30:600-607.

7. Schwarz F, Nance JW Jr, Ruzsics B, Bastarrika G, Sterzik A, Schoepf UJ. Quantification of coronary artery calcium on the basis of dual-energy coronary CT angiography. Radiology. 2012;264:700-707.

8. Fuchs TA, Stehli J, Fiechter M, et al. First experience with monochromatic coronary computed tomography angiography from a 64-slice CT scanner with gemstone spectral imaging (GSI). J Cardiovasc Comput Tomogr. 2013;7:25-31.

9. Buechel RR, Herzog BA, Husmann L, et al. Ultrafast nuclear myocardial perfusion imaging on a new gamma camera with semiconductor detector technique: first clinical validation. Eur J Nucl Med Mol Imaging. 2010;37:773-778.

10. Fiechter M, Ghadri JR, Kuest SM, et al. Nuclear myocardial perfusion imaging with a novel cadmium-zinc-telluride detector SPECT/CT device: first validation versus invasive coronary angiography. Eur J Nucl Med Mol Imaging. 2011;38: 2025-2030.

11. Herzog BA, Buechel RR, Katz R, et al. Nuclear myocardial perfusion imaging with a cadmium-zinc-telluride detector technique: optimized protocol for scan time reduction. $J$ Nucl Med. 2010;51:46-51.

12. Xu Y, Hayes S, Ali I, et al. Automatic and visual reproducibility of perfusion and function measures for myocardial perfusion SPECT. J Nucl Cardiol. 2010;17: 1050-1057.

13. Herzog BA, Buechel RR, Husmann L, et al. Validation of CT attenuation correction for high-speed myocardial perfusion imaging using a novel cadmium-zinctelluride detector technique. J Nucl Med. 2010;51:1539-1544.

14. Schepis T, Gaemperli O, Koepfli P, et al. Use of coronary calcium score scans from stand-alone multislice computed tomography for attenuation correction of myocardial perfusion SPECT. Eur J Nucl Med Mol Imaging. 2007;34: 11-19.

15. Pazhenkottil AP, Husmann L, Buechel RR, et al. Validation of a new contrast material protocol adapted to body surface area for optimized low-dose CT coronary angiography with prospective ECG-triggering. Int J Cardiovasc Imaging. 2010;26:591-597.

16. Mendonca PRS, Bhotika R, Maddah M, et al. Multi-material decomposition of spectral CT images. In: Samei E, Pelc NJ, eds. Medical Imaging 2010: Physics of Medical Imaging. Bellingham, WA: SPIE; 2010:7622-7667.

17. Buechel RR, Husmann L, Herzog BA, et al. Low-dose computed tomography coronary angiography with prospective electrocardiogram triggering. J Am Coll Cardiol. 2011;57:332-336.

18. Husmann L, Valenta I, Gaemperli O, et al. Feasibility of low-dose coronary CT angiography: first experience with prospective ECG-gating. Eur Heart J. 2008; 29:191-197.

19. Bocher M, Balan A, Krausz Y, et al. Gamma camera-mounted anatomical X-ray tomography: technology, system characteristics and first images. Eur J Nucl Med. 2000;27:619-627. 